\title{
Omega 3 fatty acids intake versus diclofenac in osteoarthritis induced in experimental rats
}

\author{
Mohammed M. El-Seweidy*1, Sousou I. Ali ${ }^{1}$, Sahar E. Elsweify ${ }^{1}$, \\ Abdelmoneim A. Ali ${ }^{2}$, and Mai M. Mashhour ${ }^{1}$
}

${ }^{1}$ Biochemistry Department, Faculty of Pharmacy, ${ }^{2}$ Pathology Department, Faculty of Veterinary medicine, Zagazig University, Zagazig, 44519, Egypt

Corresponding author: Mohammed M. El-Seweidy, Biochemistry Department, Faculty of Pharmacy, Zagazig University, Zagazig, 44519, Egypt

Submission Date: February 13th, 2017, Acceptance Date: April 27 ${ }^{\text {th }}$, 2017, Publication Date: April 30th, 2017

Citation: El-Seweidy M.M., Ali S.I., Elsweify S.E., Ali A.A., Mashhour M.M. Omega 3 fatty acids intake versus diclofenac in osteoarthritis induced in experimental rats. Functional Foods in Health and Disease 2017; 7(4); 291-302

\begin{abstract}
Background: Osteoarthritis (OA) is a degenerative joint disease, characterized by abnormal remodeling pattern of joints driven by inflammatory mediators within the affected joints. Its symptoms are many like pain, stiffness, and decreased function.
\end{abstract}

Objective: The present study mainly focused on the anti-inflammatory effect of omega 3 fatty acids (F.As) versus diclofenac, non-steroidal anti-inflammatory drug in OA induced in rats

Design: Intraarticular injection of monosodiumiodoacetate (MIA) $24.6 \mathrm{mg} / \mathrm{kg}$ in $0.6 \mathrm{ml}$ saline was used to induce OA. Diclofenacand omega-3 F. These were administered orally, daily for 21 days and after 24 hours of $\mathrm{OA}$ induction.

Results: Osteoarthritis induction resulted in an increase in serum levels of IL-6 (479.5\%), TNF$\alpha(545.5 \%)$, and CRP (754.2\%) along with IL-10 level decrease (70.3\%) as compared to normal group. Diclofenac intake demonstrated significant increase of IL-6 (24.9\%), CRP (88.6\%), and TNF- $\alpha(25.2 \%)$ compared to the OA control group. Omega 3 FAs intake showed significant reduction in inflammatory markers along with IL-10 increase, in comparison to OA group. Both treatment demonstrated a significant increase in TIMP2 along with decreased MMP2 and MPO in comparison with OA control. Positive correlation of IL-6 with MPO ( $\mathrm{r}=0.7, \mathrm{P}=0.002)$, and 
negative one with IL-10 ( $\mathrm{r}=0.9, \mathrm{p}<0.0001)$ and TIMP2 $(\mathrm{r}=-0.5, \mathrm{p}<0.008)$ was observed. Interleukin-10 was negatively correlated with MMP2 $(\mathrm{r}=-0.5, \mathrm{p}<0.007)$ and MPO $(\mathrm{r}=-0.8$, $\mathrm{p}<0.0001)$.

Conclusion: Data derived from biochemical and histopathological results, indicated that omega3 FAs may be expressed as a natural anti-inflammatory agent of a significant potential in OA with evident remarkable effect.

Keywords: OA; omega3FAs; diclofenac; MMP2; TIMP2; MPO

\section{INTRODUCTION}

Osteoarthritis (OA) is a degenerative joint disease, characterized by abnormal remodeling pattern of joints driven by inflammatory mediators within the affected joint [1,2]. Its symptoms are many like pain, stiffness, and decreased function [3]. The chondrocytes are specialized cells which consists mainly of collagen and proteoglycan, mainly acting to produce and maintain the extracellular matrix of cartilage [4], their activation in OA, is characterized by cluster formation, cell proliferation, elevated production of matrix-degrading enzymes, and matrix protein [5]. The matrix degrading enzymes involved in osteoarthritic joint includes collagenases and aggrecanases, which are members of matrix metalloproteinase (MMP) family [6]. Chondrocytes in osteoarthritic cartilage can express cytokines, chemokine receptors, and MMPs which modulate or enhance catabolic and inflammatory responses [5].

Steinbeck et al. (2007) hypothesized that the product of neutrophils and macrophages, which is myeloperoxidase enzyme (MPO), is responsible for the production of chlorine gas and highly reactive hypochlorous acid may be present in synovial fluid of OA patients [7].

Diclofenac, a well-known non-steroidal anti-inflammatory drug (NSAID), represents an inhibitor for cyclooxygenase activity and prostaglandin production. Its ability to ameliorate pain doesn't prevent the progression of symptoms of rheumatoid arthritis [8]. Cyclooxygenase_enzyme (cox) officially known as prostaglandin endoperoxide synthase which is responsible for the formation of prostanoids, including prostacycline and thromboxane. Agents which inhibit this enzyme like NSAIDS and aspirin can relieve pain and inflammation symptoms [9]. Gastrointestinal damage caused by these drugs represents a significant clinical problem and marked side effects usually observed [10]. Lastly, an increased risk of adverse cardiovascular effects was also reported during diclofenac use [11]. Therefore, searching for alternative antiinflammatory agents and effective drugs became reliable.

Omega 3 fatty acids (FAs), are essential long chain polyunsaturated FAs, including eicosapentaenoic acid (EPA) and docosahexaenoic acid (DHA), and are considered to be a dietary supplement with great benefits, including anti-inflammatory effects, reduction of coronary artery problems, and prevention of cognitive functions in those with very mild Alzheimer's disease [12]. Supplementation of omega 3 FAs have been found effective for treating (OA) and rheumatoid arthritis [13]. Additionally, such acids can initiate anti-inflammatory compounds (resolvines) through pathways involving cyclooxygenase and lipooxygenase enzymes [14]. These essential FAs are necessary for human health. In recent years, many research studies have indicated its 
valuable effect to reduce inflammation and risk of chronic diseases including cancer and arthritis. Deficiency symptoms of omega-3 fatty acids may include depression. [15, 16, 17].

Therefore, this study aims to focus more on the potential of omega 3 FAs (natural product) versus diclofenac (synthetic) in OA induced in experimental rats.

\section{MATERIAL AND METHODS}

Animals: Twenty-eight male albino rats weighing (150-180g) were used in this study. These rats were kept in plastic cages at room temperature on a 12 hours-light-dark cycle. Free access of water and rodent chow were given ad libtium.

Experimental protocol and animal handling were performed according to the guidelines of the ethical committee of the Faculty of Pharmacy, Zagazig University, Zagazig, Egypt (p5-3-2014).

\section{Induction of (OA):}

First trial to induce OA through using monosodium iodoacetate (MIA) in a dose level $12.3 \mathrm{mg} / \mathrm{kg}$ dissolved in $25 \mu \mathrm{l}$ saline for each $1 \mathrm{mg}$ of MIA[18] was found to be insufficient and was adjusted later to be $24.6 \mathrm{mg} / \mathrm{kg}$. For this reason, rats were anesthetized with thiopental (Epico Egypt) 40 $\mathrm{mg} / \mathrm{kg}$ [19]. The right knees of the rats were fixed at $90{ }^{\circ}$ shaved, and disinfected. Fresh MIA solution was injected into the joint cavity using a 26-gauge needle (single intraarticular injection). Induction of $\mathrm{OA}$ was followed by measuring the knees diameter (as inflammatory marker) using a sliding caliper.

One day after MIA injection, the rats were divided into three groups. The first one received no treatment (only MIA) and was referred to as OA control. The second one received diclofenac (Novartis Pharma.AG Basel Switzerland) $5 \mathrm{mg} / \mathrm{kg}$ body weight [20] daily for 21 days. The third group received omega 3 FAs (Arab gelatin and pharmaceutical products, Cairo, Egypt) high quality, in a dose level $1 \mathrm{~g} / \mathrm{kg}$ body weight daily for 21 days. Another group received no MIA, no treatment at all, and served as normal group.

\section{Blood and Tissue Sampling}

After 1 day of OA induction (MIA injection) 2mlblood samples were collected from all rats through retro-orbital vein and left to clot at room temperature, centrifuged and serum was collected. After 21 days, the rats were killed by retro-orbital bleeding, blood was collected for serum, and right knee joints and subchondral bones were dissected and rinsed in ice-cold saline. Part of each group (joint and bone) was fixed in $10 \%$ formalin saline for pathological examination. The rest of joints were frozen at $-80^{\circ} \mathrm{C}$ for subsequent biochemical measurements of MPO, MMP2, and TIMP2.

\section{Determination of serum markers:}

Interleukin-6(IL-6), IL-10, tumor necrosis factor $\alpha$ (TNF- $\alpha$ ) and C-reactive protein (CRP) were determined using ELISA technique kits as reported by Banerjee et al., (2003) [21] (Ray Biotech Inc., DRG International Inc., USA, Koma Biotech. Inc., Korea and BD Biosciences) respectively, and we have followed the recommendations of the manufacturers.

\section{RT-PCR measurement of tissue MMP2, TIMP2, and MPO gene expression analysis:}


Matrix metalloproteinase 2, TIMP2, and MPO were detected in the knee joints by RT-PCR technique [22]. Total RNA was extracted from joint tissue samples using SV Total RNA isolation system (Promega, Madison, WI, USA) which provides fast and simple technique for isolation of RNA.SV mean (spin or vacuum) purification protocol. The extracted RNA was reverse transcribed into cDNA and amplified by PCR using RT-PCR kit (Stratagene, USA). Reactions were performed in a $50 \mu 1$ final volume $(25 \mu 1$ SYBR Green Mix $(2 x), 0.5 \mu 1$ cDNA, $2 \mu 1$ primer pair mix ( $5 \mathrm{pmol} /$ $\mu 1$ each primer), $22.5 \mu 1 \mathrm{H} 2 \mathrm{O}$ ). Each PCR run was performed under the following conditions: $50^{\circ} \mathrm{C}$ for $2 \mathrm{~min}(1 \mathrm{cycle}), 95^{\circ} \mathrm{C}$ for $10 \mathrm{~min}(1 \mathrm{cycle}), 95^{\circ} \mathrm{C}$ for $15 \mathrm{sec}, 60^{\circ} \mathrm{C}$ for $30 \mathrm{sec}$, and $72^{\circ} \mathrm{C}$ for 30 sec $\left(40\right.$ cycles) then $72^{\circ} \mathrm{C}$ for $10 \mathrm{~min}(1 \mathrm{cycle})$ followed by a single fluorescence measurement. Relative gene expression was calculated using the $2-\Delta \Delta \mathrm{Ct}$ method.

MMP2:

Forward primer: 5'TATAGAACTCCAACCACAC-3'

Reverse primer: 5'CCGTAGAAGGTCCTCGTC-3'

TIMP2:

Forward primer: 5'-CGCTACCAACGGTGGAAGRC-3'

Reverse primer: 5'-CAAGCTCCCCCTCTTTTTCA-3'

MPO:

Forward primer: 5'-ACCTACCCCAGTACCGATCC-3'

Reverse primer: 5'-AACTCTCCAGCTGGCAAAAA-3'

\section{Histopathological study:}

Right knee joints and subchondral bones were kept in $10 \%$ buffered formalin and $1 \% \mathrm{HNO}_{3}$ for 1 day or more till they become soft, rinsed with runny water then put in alcohol series (different concentrations of alcohol) in order to remove all water from tissues before putting in paraffin which is hydrophobic), and were kept in xylene paraffin. Leica Microtome was used to cut fivemicron tissue sections, stained with haematoxylin and eosin (H \& E) for histopathological examination. Table 4: is a table for knowing to what score or grade the OA arrived. Score zero: normal (no OA), then as the score increases the OA injury increases, the last score (5) mean that no cartilage exist and bones fused.

\section{STATISTICAL ANALYSES}

All statistical analyses were performed using Prism 5', Graph Pad,CA, USA, Program. Results were presented as mean \pm SD. Statistical difference were determined using Student's t-test, one way analysis of variance followed by Tukey test, $\mathrm{P}<0.05$ is considered statistically significant.

\section{RESULTS AND DISCUSSION}

Intraarticular injection of MIA induced an inflammatory state, characterized by an increase of the diameter of the knees after 1 day and turned to be decreased. 
Osteoarthritis induction resulted in an increase in serum levels of IL-6 (479.5\%), TNF- $\alpha$ (545.5\%), and CRP (754.2\%) along with an IL-10 level decrease (70.3\%) compared to the normal group (Tab.1).

Table 1: Serum inflammatory and anti-inflammatory markers after MIA injection (OA induction).

\begin{tabular}{|c|c|c|}
\hline Parameters & Normal group & OA group \\
\hline IL-6 $(\mathrm{pg} / \mathrm{ml})$ & $8.3 \pm 1.1$ & $48.1 \pm 4.7^{*}$ \\
\hline IL-10 $(\mathrm{pg} / \mathrm{ml})$ & $57.9 \pm 3.3$ & $17.2 \pm 1.5^{*}$ \\
\hline TNF- $\alpha(\mathrm{pg} / \mathrm{ml})$ & $11.2 \pm 1$ & $72.3 \pm 5.5^{*}$ \\
\hline CRP $(\mathrm{ng} / \mathrm{ml})$ & $2.4 \pm 0.6$ & $20.5 \pm 2.2^{*}$ \\
\hline
\end{tabular}

Values are presented as mean $\pm \mathrm{SD}, \mathrm{n}=7, \mathrm{P}<0.05$

*Significant results versus normal group.

Diclofenac intake demonstrated significant increase of ofIL-6 (24.9\%), CRP (88.6\%), and TNF- $\alpha(25.2 \%)$ as compared to the OA control group. Omega 3 FA intake showed significant reduction in inflammatory markers along with IL-10 increase, in comparison to OA group (Fig.1).
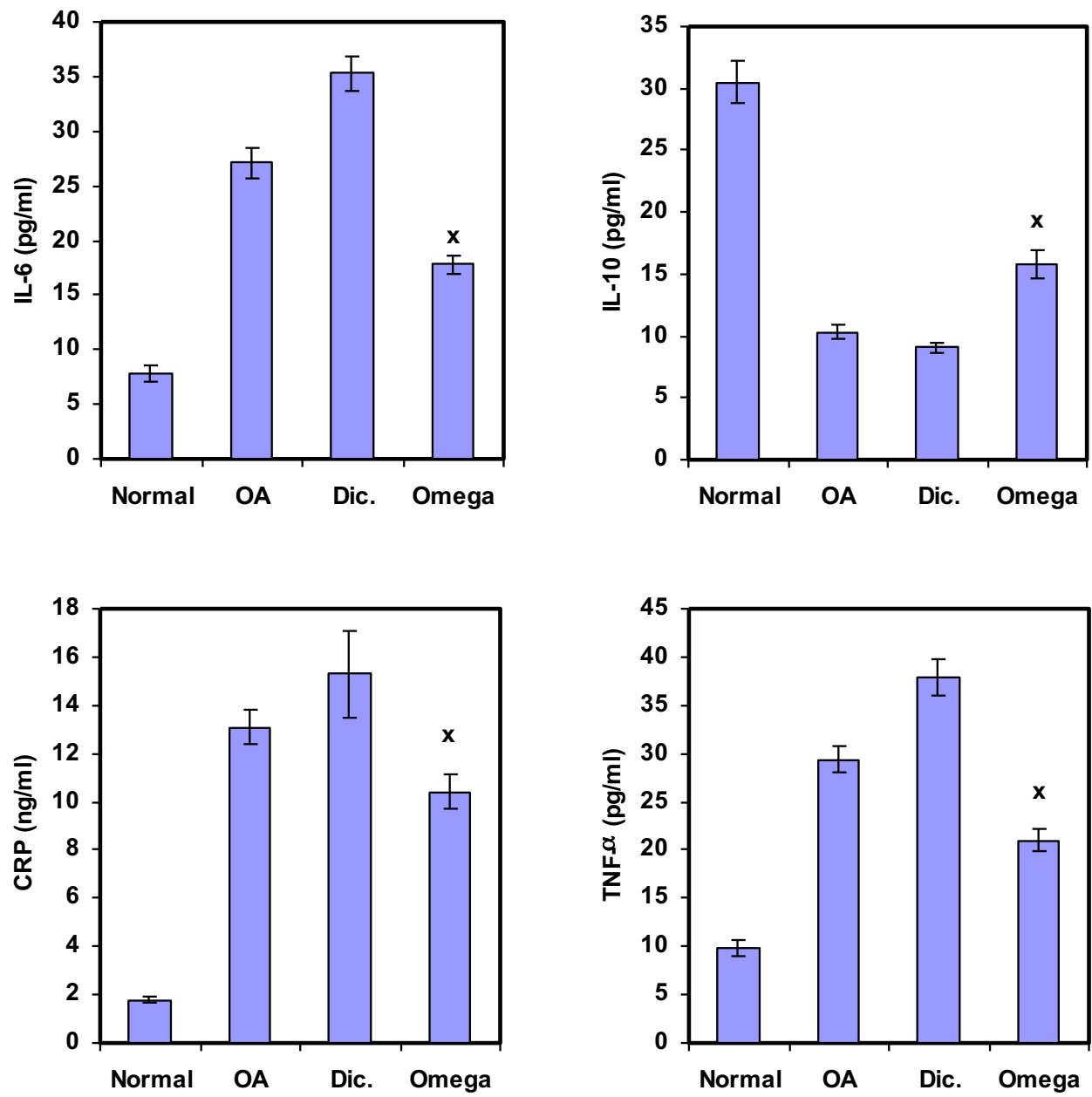

Fig. 1: Effect of omega-3 FAs versus diclofenac on serum levels of inflammatory markers and anti-inflammatory one following 21 days of treatment. 
Values are presented as mean \pm SD, $n=7, \mathrm{p}<0.05$

$\mathrm{x}$ : significant effect versus diclofenac group

Both treatment demonstrated a significant increase in TIMP2 along with decreased MMP2 and MPO in comparison with OA control (Tab. 2).

Table 2: Tissues MPO, MMP2, and TIMP2 of diclofenac and omega3 FAs groups in comparison to OA control

\begin{tabular}{|c|c|c|c|c|}
\hline Parameter & Normal & $\begin{array}{c}\text { OA } \\
\text { Control }\end{array}$ & $\begin{array}{l}\text { Diclofenac } \\
\text { group }\end{array}$ & $\begin{array}{r}\text { Omega3 FAs } \\
\text { group }\end{array}$ \\
\hline MPO/GADPH & $0.1 \pm 0.02$ & $0.9 \pm 0.1^{*}$ & $0.6 \pm 0.1^{*}$ & $0.6 \pm 0.1^{*}$ \\
\hline MMP2/GADPH & $1.2 \pm 0.2$ & $10.3 \pm 1.8^{*}$ & $2.4 \pm 0.3^{*}$ & $3.9 \pm 0.6^{*}$ \\
\hline TIMP2/GADPH & $25.5 \pm 4$ & $11.5 \pm 2.7^{*}$ & $20 \pm 2.3^{*}$ & $21.2 \pm 4$ \\
\hline
\end{tabular}

Values are represented as mean $\pm \mathrm{SD}, \mathrm{n}=7, \mathrm{P}<0.05$

* Significant from normal group.

- Significant from MIA induced OA

Positive correlation of IL-6 with MPO ( $\mathrm{r}=0.7, \mathrm{P}=0.002)$, and negative one with IL-10( $\mathrm{r}=-0.9$, $\mathrm{p}<0.0001)$ and TIMP2 $(\mathrm{r}=-0.5, \mathrm{p}<0.008)$ were observed. Interleukin- 10 was negatively correlated with MMP2(r=- 0.5, $\mathrm{p}<0.007)$ and MPO $(\mathrm{r}=-0.8, \mathrm{p}<0.0001)(\mathrm{Tab} .3)$.

Table 3: Correlation coefficient between selected markers.

\begin{tabular}{|c|c|c|r|r|r|r|r|c|}
\hline \multirow{2}{*}{ Item } & \multicolumn{2}{|c|}{ MPO } & \multicolumn{2}{|c|}{ MMP2 } & \multicolumn{2}{|c|}{ TTMP2 } & \multicolumn{2}{|c|}{ IL-10 } \\
\cline { 2 - 9 } & $\mathrm{R}$ & $\mathrm{P}$ & $\mathrm{R}$ & $\mathrm{P}$ & $\mathrm{R}$ & $\mathrm{P}$ & $\mathrm{R}$ & $\mathrm{P}$ \\
\hline IL-6 & 0.7 & 0.0002 & - & - & -0.5 & 0.008 & -0.9 & $<0.0001$ \\
\hline MPO & & & & & -0.8 & $<0.0001$ & -0.8 & $<0.0001$ \\
\hline TNF $\alpha$ & 0.7 & 0.003 & - & - & -0.5 & 0.01 & & \\
\hline
\end{tabular}

Histopathological examination of osteoarthritic joints showed pyknotic chondrocytes, disorganized lacunae and fragmentation of bony trabeculae replaced by fibrous tissues, numerous osteoclasts and inflammatory cells (score 4 table4) (fig 2 B,C). Diclofenac group showed hyperplasia of synovial membrane, hypertrophy, and little edema (score 2 table 4) (fig2 D). Omega3 FAs on the other hand showed atrophy of articular surface, disorganized lacunae and 
pyknotic chondrocytes (score 3) (fig $2 \mathrm{E}$ ) in a conclusion that both drugs have exerted certain improvement in histopathology and not total recovery as compared to OA control (Fig.2).

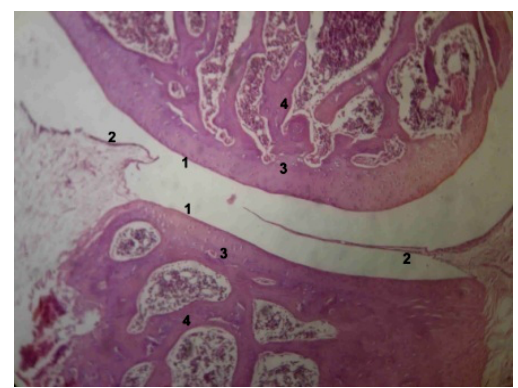

(A)

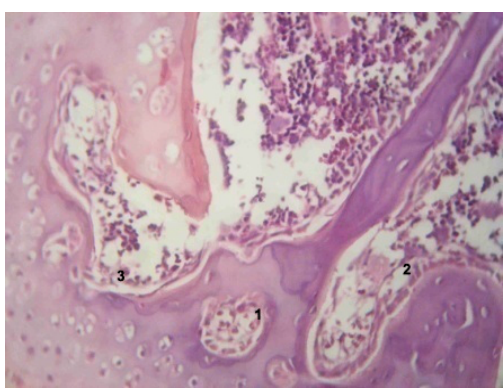

(B)

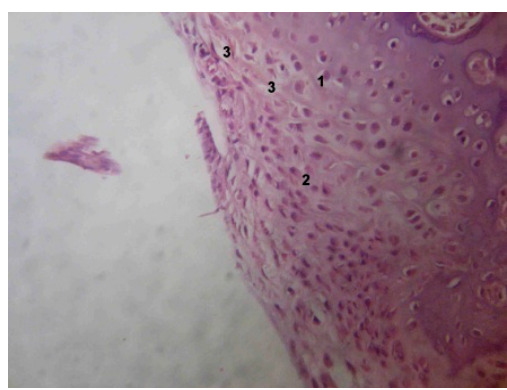

(C)

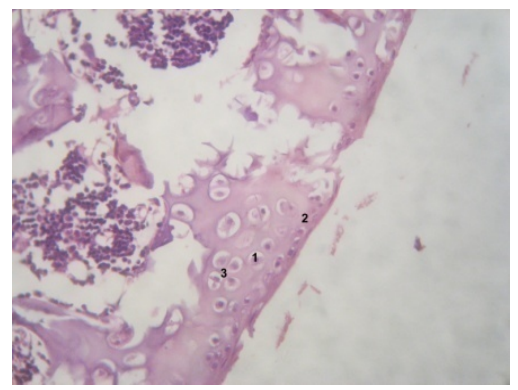

(E)

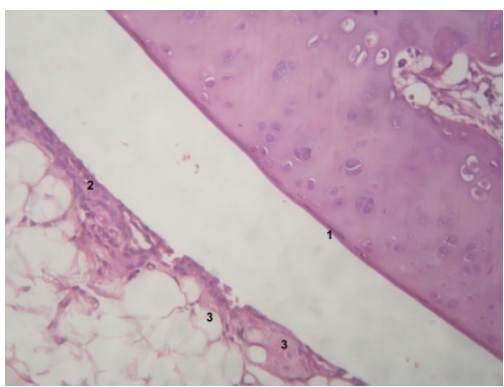

(D)

Fig. 2: Photomicrograph of knee joints of normal (H \& E, X 300), osteoarthritic and osteoarthritic treated rats (H \& E, X 1200).

(A) Normal knee showed normal articular surface ${ }^{1}$, synovium $^{2}$, chondrocyte $^{3} \&$ bone $^{4}$.

(B) Osteoarthritic knee showed pyknotic chondrocytes ${ }^{1}$, disorganized laucnae ${ }^{2}$ and lysis of matrix proteoglycan with little debris ${ }^{3}$ inside articualr cavity.

(C) Osteoarthritis knee showed fragmentation of bony trabecube replaced by fibrous tissue ${ }^{1}$, numerous osteoclast ${ }^{2}$ and inflammatory cell ${ }^{3}$.

(D) Diclofenac treated group: Normal articular surface ${ }^{1}$, synovial membrane hyperplasia ${ }^{2}$ and hypertrophy ${ }^{3}$, little edema ${ }^{4}$.

(E) Omega 3 FAs treated group: Pyknotic chondrocytes ${ }^{1}$, atrophy of articualr surface ${ }^{2}$ and disorganized lacunae ${ }^{3}$.

Table 4: Scoring for OA lesions

\begin{tabular}{|l|c|}
\hline \multicolumn{1}{|c|}{ Lesions } & Score \\
\hline - Normal articular surface, synovium, and bone & 0 \\
\hline - Degeneration of cartilage with loss of proteoglycan. & 1 \\
\hline - Synovial membrane edema. & 2 \\
\hline - Secrosis of chondrocytes or erosion of articular surface. & \\
\hline - Atrophy of articular surface with diorganized lacunae. & 3 \\
\hline - Loss of cartilage, subchondral bone ulcer. & 4 \\
\hline
\end{tabular}


- Chronic inflammatory cells and fibrosis of synovial membrane.

- Sclerosis of subchondral bone.

- Ankylosis (bone fusion).

In the present work, we used OA induced model, developed by intraarticular injection of MIA. Guzman et al. (2003) indicated that MIA produced arapid straightforward model that mimic the pathological and pharmacological features of human OA [23]. Monosodium iodoacetateis expressed as an inhibitor of glycolytic pathway, leading to chondrocytes death through blocking glyceraldehyde 3-phosphate dehydrogenase activity and metabolic disruption [24]. It is expressed also as an inducer for chemical injury along with inflammation of synovial membrane or chondrocytes [25]. This is in consistence with fig 2(B,C).

Loeser (2006) indicated that inflammation of synovium contributes to disregulation of chondrocyte function leading to an imbalance between anabolic and catabolic activities of chondrocytes. The activated synovial membrane of chondrocytes in turn represents the main source of the pro-inflammatory mediators increase. Tumor necrosis- factor $\alpha$ produced from synovial membrane can also induce IL-6 up-regulation [26]. The present study, in agreement, demonstrated significant increase in IL- 6 and TNF- $\alpha$ levels in subsequent to MIA administration.

Myeloperoxidase increases in the present study in subsequent to oxidative stress observed, may be due to the presence of neutrophils and/or macrophages in osteoarthritis joints[27].

Current data revealed remarkable increase in MMP2 along with TIMP2 decrease in osteoarthritis rats, which is in agreement with previous study [28]. This may be attributed to certain imbalance occurred between the two enzymes and reflecting a high rate of turnover in articular cartilage [29]. Iannone et al. (2001) also explained that TNF- $\alpha$ can drive the catabolic process in OA leading to cartilage degradation [30].

Significant increase in serum IL-6, TNF- $\alpha$, and CRP was observed in diclofenac group as compared to OA control.

Subramanian (2009) considered diclofenac as a toxic drug inducing cell injury, mostly attributed to its ability for binding of macromolecules in situation having low intracellular quantities of NADH, NADPH, and GSH [31].

Elevation in TNF- $\alpha$ following diclofenac administration in the present study may be attributed to PG2 decrease and could exacerbate the pro-inflammatory environment within the rheumatoid arthritis joints and the systemic environment [8].

Paino et al. (2005) indicated that therapeutic plasma level of diclofenac is able to suppress the oxidative functions of neutrophils by inhibiting MPO chlorinating activity and also by scavenging hypochlorous acid [32]. This may explain the decreased level of MPO in diclofenac group of the present study.

Interestingly, MPO has been found before to disrupt the physiological balance of the activity of MMPs and functions of MMP inhibitors in inflammation state [33]. Negative correlation between MPO and both TIMP2 and IL-10 were observed and in agreement with the last study.

Omega 3 FAs treatment significantly attenuated the inflammatory markers (IL-6, TNF- $\alpha$ and CRP), along with IL-10 increase as compared to OA control. This is in consistence with other studies [17]. 
Supplementation of omega3FAs can reduce serum soluble TNF $\alpha$ receptor $\mathrm{p} 55$ and CRP in rheumatoid arthritis patients [34], the production of pro-inflammatory cytokines and other proinflammatory proteinsase mainly induced via the NFKB system [35].

Reduction of cytokines levels and CRP following omega3FAs administration may involve both peroxisome proliferator agonist PPAR and nuclear factor $\mathrm{\kappa B}(\mathrm{NF}-\mathrm{\kappa B})$ mechanisms, omega3 FAs are considered as natural ligand of PPAR [36]. The latter was a ligand activated transcription factor. The complex including PPAR and its ligand will bind to peroxisome proliferator response element on DNA to regulate gene expression of these markers [37]. Peroxisome proliferator agonist inhibited the activation of (NF-KB) [38], indeed gene encoding for TNF- $\alpha$ and IL-6 [39].

Omega 3FAs potentiated also the anti-inflammatory effect as observed in IL-10 increase, mostly attributed to the expression and secretion of IL-10 in human monocytes through PPAR $\gamma$ pathway[40].

Curtis et al. (2002) indicated that fish oil (omega3 FAs) reduced MMP and increased TIMP2 production as observed in the present study via both TNF- $\alpha$ and prostaglandin pathways[41].

The present study can express that omega 3FAs have anantidegradation along with antioxidant effects as exerted by TIMP2 increase along with MPO and MMP2 decrease.

\section{CONCLUSION}

Tissue biomarkers and histopathological profiles of diclofenac and omega3FAs groups showed to a certain extent similar patterns. However, omega3 FAs group demonstrated marked antiinflammatory effect as compared to the diclofenac group. This in turn may support the use of the first (natural product) as a therapeutic alternative. However, clinical study may be recommended to confirm the experimental rat results.

List of abbreviations: Fatty acids (F.As),osteoarthritis(OA), monosodiumiodoacetate (MIA),myeloperoxidase (MPO), tissue inhibitor metalloproteinase (TIMP2), matrix metalloproteinase (MMP), non-steroidal anti-inflammatory drug (NSAID), epicosapentaenoic acid( EPA), docosahexaenoic acid (DHA), interleukine-6 (IL-6), tumor necrosis factor $\alpha$ (TNF- $\alpha$ ), C-reactive protein (CRP) and nuclear factor $\kappa \mathrm{B}(\mathrm{NF}-\mathrm{\kappa B})$.

Competing interest: The authors have no financial interest or conflicts of interest.

Author's contribution: All authors contributed to this work.

Acknowledgement: The histopathological work of the current study was kindly performed by Dr. Heba Khalil Mohammad, Theodor Belhars Research Institute Egypt.

\section{REFERENCES}

1. Felson, D.T., et al., Osteoarthritis: new insights. Part 1: the disease and its risk factors. Annals of internal medicine, 2000. 133(8): p. 635-46.

2. Blagojevic, M., et al., Risk factors for onset of osteoarthritis of the knee in older adults: a systematic review and meta-analysis. Osteoarthritis and cartilage, 2010. 18(1): p. 24-33. 
3. Farid, R., et al., Oral intake of purple passion fruit peel extract reduces pain and stiffness and improves physical function in adult patients with knee osteoarthritis. Nutrition research, 2010. 30(9): p. 601-6.

4. Eyre, D. Collagen of articular cartilage. Arthritis Res. 2002, 4, 30-35.

5. Goldring, M.B. and K.B. Marcu, Cartilage homeostasis in health and rheumatic diseases. Arthritis research \& therapy, 2009. 11(3): p. 224.

6. Troeberg, L. and H. Nagase, Proteases involved in cartilage matrix degradation in osteoarthritis. Biochimica et biophysica acta, 2012. 1824(1): p. 133-45.

7. Steinbeck, M.J., et al., Myeloperoxidase and chlorinated peptides in osteoarthritis: potential biomarkers of the disease. Journal of orthopaedic research : official publication of the Orthopaedic Research Society, 2007. 25(9): p. 1128-35.

8. Page, T.H., et al., Nonsteroidal anti-inflammatory drugs increase TNF production in rheumatoid synovial membrane cultures and whole blood. Journal of immunology, 2010. 185(6): p. 3694-701.

9. Fitzpatrick, F.A., Cyclooxygenase enzymes: regulation and function.Curr Pharm Des. 2004;10(6):577-88 .

10. Wallace, J.L., et al., Gastrointestinal safety and anti-inflammatory effects of a hydrogen sulfide-releasing diclofenac derivative in the rat. Gastroenterology, 2007. 132(1): p. 26171.

11. McGettigan, P. and D. Henry, Cardiovascular risk with non-steroidal anti-inflammatory drugs: systematic review of population-based controlled observational studies. PLoS medicine, 2011. 8(9): p. e1001098.

12. Swanson, D., R. Block, and S.A. Mousa, Omega-3 fatty acids EPA and DHA: health benefits throughout life. Advances in nutrition, 2012. 3(1): p. 1-7.

13. Cleland, L.G., M.J. James, and S.M. Proudman, The role of fish oils in the treatment of rheumatoid arthritis. Drugs, 2003. 63(9): p. 845-53.

14. Serhan, C.N., N. Chiang, and T.E. Van Dyke, Resolving inflammation: dual antiinflammatory and pro-resolution lipid mediators. Nature reviews. Immunology, 2008. 8(5): p. 349-61.

15. Assisi A., R. Banzi, C. Buonocore, et al. Fish oil and mental health: the role of n-3 longchain polyunsaturated fatty acids in cognitive development and neurological disorders. IntClinPsychopharmacol. 2006 Nov;21(6):319-36

16. Frangou S., M. Lewis, PMcCrone,Efficacy of ethyl-eicosapentaenoic acid in bipolar depression: randomised double-blind placebo-controlled study. Br J Psychiatry. 2006 Jan; 188:46-50

17. Elseweidy M.M., S.A.Ibrahim , S.E.Elswefy and M. M.Mashhour,Omega-3 fatty acids , atorvastatin as modulator for inflammatory pattern versus Diclofenac in osteoarthritis induced in experimental rats.African journal of pharmacy and pharmacology,2016.10(22):472-479

18. Bar-Yehuda, S., et al., Induction of an antiinflammatory effect and prevention of cartilage damage in rat knee osteoarthritis by CF101 treatment. Arthritis and rheumatism, 2009. 60(10): p. 3061-71. 
19. Hildebrandt, I.J., H. Su, and W.A. Weber, Anesthesia and other considerations for in vivo imaging of small animals. ILAR journal, 2008. 49(1): p. 17-26.

20. Wahane, V.D. and V.L. Kumar, Atorvastatin ameliorates inflammatory hyperalgesia in rat model of monoarticular arthritis. Pharmacological research, 2010. 61(4): p. 329-33.

21. Banerjee, M., et al., Modulation of inflammatory mediators by ibuprofen and curcumin treatment during chronic inflammation in rat. Immunopharmacology and immunotoxicology, 2003. 25(2): p. 213-24.

22. Pfaffl, M.W., A new mathematical model for relative quantification in real-time RT-PCR. Nucleic acids research, 2001. 29(9): p. e45.

23. Guzman, R.E., et al., Mono-iodoacetate-induced histologic changes in subchondral bone and articular cartilage of rat femorotibial joints: an animal model of osteoarthritis. Toxicologic pathology, 2003. 31(6): p. 619-24.

24. Grossin, L., et al., Gene transfer with HSP 70 in rat chondrocytes confers cytoprotection in vitro and during experimental osteoarthritis. FASEB journal : official publication of the Federation of American Societies for Experimental Biology, 2006. 20(1): p. 65-75.

25. Goldring, M.B. and S.R. Goldring, Osteoarthritis. Journal of cellular physiology, 2007. 213(3): p. 626-34.

26. Loeser, R.F., Molecular mechanisms of cartilage destruction: mechanics, inflammatory mediators, and aging collide. Arthritis and rheumatism, 2006. 54(5): p. 1357-60.

27. Bonnet, C.S. and D.A. Walsh, Osteoarthritis, angiogenesis and inflammation. Rheumatology, 2005. 44(1): p. 7-16.

28. Kim, J.K., et al., Effect of GCSB-5, a Herbal Formulation, on Monosodium IodoacetateInduced Osteoarthritis in Rats. Evidence-based complementary and alternative medicine : eCAM, 2012. 2012: p. 730907.

29. Alam, M.R., et al., Biomarkers for identifying the early phases of osteoarthritis secondary to medial patellar luxation in dogs. Journal of veterinary science, 2011. 12(3): p. 273-80.

30. Iannone, F., et al., Interleukin-10 and interleukin-10 receptor in human osteoarthritic and healthy chondrocytes. Clinical and experimental rheumatology, 2001. 19(2): p. 139-45.

31. Subramanian S., Diclofenac induced toxic manifestations on adjuvant induced arthritic rats pheripheral and reproductive organ of male wistar rats rattusnorvegicus. J Toxicol Environ Health Sci, 2009. 1(1): p. 012-021.

32. Paino, I.M., et al., Effect of therapeutic plasma concentrations of non-steroidal antiinflammatory drugs on the production of reactive oxygen species by activated rat neutrophils. Brazilian journal of medical and biological research $=$ Revista brasileira de pesquisas medicas e biologicas, 2005. 38(4): p. 543-51.

33. Mainnemare, A., et al., Hypochlorous acid and taurine-N-monochloramine in periodontal diseases. Journal of dental research, 2004. 83(11): p. 823-31.

34. Moghaddami, N., et al., Novel action of n-3 polyunsaturated fatty acids: inhibition of arachidonic acid-induced increase in tumor necrosis factor receptor expression on neutrophils and a role for proteases. Arthritis and rheumatism, 2007. 56(3): p. 799-808.

35. Calder, P.C., Omega-3 fatty acids and inflammatory processes. Nutrients, 2010. 2(3): p. 355-74. 
36. Xu, H.E., et al., Molecular recognition of fatty acids by peroxisome proliferator-activated receptors. Molecular cell, 1999. 3(3): p. 397-403.

37. Houseknecht, K.L., B.M. Cole, and P.J. Steele, Peroxisome proliferator-activated receptor gamma (PPARgamma) and its ligands: a review. Domestic animal endocrinology, 2002. 22(1): p. 1-23.

38. Rossi, A., et al., Anti-inflammatory cyclopentenone prostaglandins are direct inhibitors of IkappaB kinase. Nature, 2000. 403(6765): p. 103-8.

39. Robinson, L.E., A.C. Buchholz, and V.C. Mazurak, Inflammation, obesity, and fatty acid metabolism: influence of n-3 polyunsaturated fatty acids on factors contributing to metabolic syndrome. Applied physiology, nutrition, and metabolism = Physiologie appliquee, nutrition et metabolisme, 2007. 32(6): p. 1008-24.

40. Satoh-Asahara, N., et al., Highly purified eicosapentaenoic acid increases interleukin-10 levels of peripheral blood monocytes in obese patients with dyslipidemia. Diabetes care, 2012. 35(12): p. 2631-9.

41. Curtis, C.L., et al., Pathologic indicators of degradation and inflammation in human osteoarthritic cartilage are abrogated by exposure to n-3 fatty acids. Arthritis and rheumatism, 2002. 46(6): p. 1544-53. 